\title{
A novel therapeutic combination of mesenchymal stem cells and stigmasterol to attenuate osteoarthritis in rodent model system - a proof of concept study
}

\author{
Samuel Joshua Pragasam Sampath, Nagasuryaprasad Kotikalapudi, Vijayalakshmi Venkatesan \\ Stem Cell Research Laboratory, Department of Biochemistry/Cell and Molecular Biology, National Institute of Nutrition (Indian Council of \\ Medical Research), Tarnaka, Hyderabad, Telangana, India \\ Correspondence to: Dr. Vijayalakshmi Venkatesan, PhD. Scientist 'G' \& Head, Stem Cell Research Division, Department of Biochemistry/Cell and \\ Molecular Biology, National Institute of Nutrition - Indian Council of Medical Research, Jamai Osmania (PO), Hyderabad, 500 007, Telangana, \\ India. Email: v.venkateshan@gmail.com.
}

\begin{abstract}
Mesenchymal stem cells (MSCs) have gained wide therapeutic acceptance in regenerative medicine due to their potential in repair process in restoring the damaged tissues and controlling inflammation. In the present study, we report for the first time the beneficial effects of combining placentalderived MSCs (hPMSCs) with stigmasterol-a plant-derived sterol to accelerate cartilage repair and regeneration in a monosodium-iodoacetate (MIA) induced osteoarthritis (OA) rat model. Control animals (Group I) received no treatment. Experimental animals (Group II) received a single intra-articular injection of MIA (2 mg) in the right knee joints. The Group II animals developed OA-like lesions within a week of MIA injection. They were subdivided further as: (II-A): OA, (II-B): OA+hPMSCs $\left(2 \times 10^{6}\right.$ cells, single-dose/ intra-articular injection), (II-C): OA+stigmasterol $(20 \mu \mathrm{g} / \mathrm{mL}$, single-dose/intra-articular injection) and (IID): OA+hPMSCs+stigmasterol. The animals were monitored for four more weeks after which they were sacrificed, the right limbs dissected out and assessed for cartilage repair and regeneration using microcomputed tomography (micro-CT) and histology. Results showed that the combined administration of hPMSCs with stigmasterol (II-D) was the most effective in correcting the OA lesions, with concomitant repair and regeneration. However, hPMSCs (II-B) or stigmasterol (II-C) per se treated groups showed only marginal beneficial effects and were not significant. Thus the present study provides valuable insights in situ using a combination of hPMSCs and stigmasterol towards cartilage repair and regeneration. We advocate the participation of populating cells or residual chondrocytes in addition to its anti-inflammatory functions.
\end{abstract}

Keywords: Mesenchymal stem cells (MSCs); stigmasterol; osteoarthritis (OA); monosodium iodoacetate; microcomputed tomography (micro-CT); histology

Received: 05 October 2020; Accepted: 10 March 2021; Published: 23 March 2021.

doi: $10.21037 /$ sci-2020-048

View this article at: http://dx.doi.org/10.21037/sci-2020-048

\section{Introduction}

Osteoarthritis (OA) is the most common joint disorder affecting millions worldwide. It is characterized by cartilage breakdown, which drives a full-blown inflammation achieved by a networking of pro-inflammatory cytokines, matrix metalloproteinases (MMPs) and aggrecanases resulting in irreversible cartilage erosion-the hallmark of OA (1).
Currently more than 50 modalities of pharmacological, non-pharmacological, and surgical treatment for OA are reported in the literature. However, they primarily aim to alleviate OA symptoms and fail to regenerate the lost cartilage and prevent disease progression (2). Regenerative techniques for articular cartilage defects offer a newer paradigm for treating OA. In recent times, mesenchymal stem cells (MSCs)-based approaches have 
gained momentum towards therapy to address diabetes, pulmonary, cardiovascular, neurological disorders and musculoskeletal diseases (bone/cartilage regeneration) (3) by virtue of their immunomodulatory, anti-inflammatory and multipotent functions (4). In the field of OA, studies have predominantly explored to address the promises of MSCs therapy in focal cartilage defects as compared to generalized cartilage loss, which is more rampant in population. This necessitates exploring alternative strategies for the effective management of generalized OA. Stigmasterol, also known as Stigmasterin or Wulzen anti-stiffness factor, is a plant-derived sterol well characterized to possess antihypercholesterolemic, anti-tumor, hypoglycaemic, antimutagenic, antioxidant, anti-inflammatory (5), and antiOA effects in vitro (6) and in vivo (7). Stigmasterol is predominantly found in fats and oils of soybean, calabar bean, rapeseed, and is involved in the synthesis of hormones like androgens, progesterone, corticoids and estrogens (8). Keeping in view of the beneficial effects of Stigmasterol and MSCs, we hypothesize that combining human placentalderived MSCs (hPMSCs) with stigmasterol would bring about enhanced tissue repair/regeneration in experimental $\mathrm{OA}$ in comparison with the individual treatments, thereby better treatment outcomes. We aimed to assess if there could be any beneficial effects by combining hPMSCs with stigmasterol to bring about amelioration of $\mathrm{OA}$ in a monosodium-iodoacetate (MIA)-induced rat model of OA using micro-CT and histopathological analysis. The MIAinduced $\mathrm{OA}$ in rats closely mimics human OA pathology wherein the MIA injection causes chondrocyte cell death leading to disorganization/degeneration of the articular cartilage resulting in $\mathrm{OA}(9)$.

\section{Methods}

\section{Animals}

All animal experiments were carried out in accordance with the guidelines of the Committee for the Purpose of Control and Supervision of Experiments on Animals (CPCSEA). The study was approved by the Institutional Animal Ethical Committee, National Institute of Nutrition, Hyderabad (P29F/III-IAEC/NIN/12/2016). Female Wistar rats weighing between 150-180 grams were obtained from the Animal Facility, NIN, housed individually in standard polypropylene cages and maintained at $22 \pm 1{ }^{\circ} \mathrm{C}$ with $12 \mathrm{~h}$ dark/light cycles and humidity of 50-60\%. They were fed standard laboratory rat chow prepared in house with free access to water.

\section{Isolation of bPMSCs, culture and characterization}

Placenta was sourced from a local maternity hospital, and informed consent was obtained from the volunteer before collecting the placenta. The hPMSCs were isolated from the placenta as per published protocols (10). Briefly, a weighed amount of tissue from the placental chorionic plate was washed in sterile phosphate-buffered saline (PBS) $\mathrm{pH} 7.2$ to remove any trace of cord blood. The tissue was finely chopped into small pieces followed by enzymatic digestion using $0.25 \%$ trypsin (Gibco, Life Technologies, Grand Island, USA) for $30 \mathrm{~min}$ at $37^{\circ} \mathrm{C}$. The digest was then centrifuged at 1,000 rpm (Eppendorf 5810R, Hamburg, Germany) for $5 \mathrm{~min}$ to separate the cells. The cells were then washed three times with PBS, and the cell pellet was suspended in Dulbecco's Modified Essential Medium/Ham's F12 (1:1) medium (DMEM/F12, Invitrogen, CA, USA) supplemented with $10 \%$ fetal bovine serum (FBS) (Gibco, Thermo Fisher, Scientific, CA, USA), penicillin (100 units/mL) and streptomycin $(100 \mu \mathrm{g} / \mathrm{mL})$ at $37{ }^{\circ} \mathrm{C}, 5 \% \mathrm{CO}_{2}$ and $95 \%$ humidity in a $\mathrm{CO}_{2}$ incubator. The hPMSCs were identified by their fibroblastic morphology. The hPMSCs at passage 3 (P3) were confirmed for their ability to differentiate into chondrogenic, osteogenic and adipogenic lineages using commercially available kits (Gibco, Life Technologies, USA). The hPMSCs were also characterized for expression of CD133 (Abcam, Cat. No. ab19898, 1:100) and STRO1 (Sigma Aldrich, Cat. No. MAB4315, 1:100) MSC specific markers by immunofluorescence (11) using Leica SP5 confocal microscope equipped with Leica Advanced Fluorescence software (Mannheim, Germany).

\section{$O A$ induction and treatment}

To induce OA, MIA was dissolved in sterile saline solution. The rats were anesthetized with $5 \%$ isoflurane, and the knees were shaved and disinfected before the injections. OA was induced in the rats with $2 \mathrm{mg}$ of MIA in a total volume of $50 \mu \mathrm{L}$ (12). MIA was injected intra-articularly through the patellar ligament of the right knee, using a 26-gauge needle, while the rat was under anesthesia. Caution was exercised to ensure that the needle was not advanced too far into the cruciate ligaments.

One week later, the MIA injected rats developed OA as evidenced by swelling of the injected knees following which 
the rats were randomly divided into five groups as per the following experimental design with six animals in each group: Group (I): controls (no injections), Group (II-A): MIA-induced OA rats [OA], Group (II-B): OA rats treated with hPMSCs $\left(2 \times 10^{6}\right.$ cells in PBS), Group (II-C): OA rats treated with stigmasterol $(20 \mu \mathrm{g} / \mathrm{mL}$ in PBS), Group (II-D): OA rats treated with a combination of hPMSCs $\left(2 \times 10^{6}\right.$ cells $)$ and stigmasterol $(20 \mu \mathrm{g} / \mathrm{mL})$. Stigmasterol was dissolved in ethanol and added at a final concentration of $20 \mu \mathrm{g} / \mathrm{mL}$ to the hPMSCs cell suspension just before the injections were given to the rats. The hPMSCs and stigmasterol were administered intra-articularly using the method mentioned earlier. MIA and stigmasterol were procured from SigmaAldrich, St. Louis, MO.

\section{Micro-CT and bistological assessment of rat knee joints}

Four weeks post the treatments, the rats were euthanized by $\mathrm{CO}_{2}$ asphyxiation, and the right hind limbs were collected and fixed in $10 \%$ neutral-buffered formalin. The hind limbs were scanned using SkyScan 1176 micro-CT system and software (SkyScan, Belgium) with pixel size $17.32 \mu \mathrm{m}$, voltage $65 \mathrm{kV}$, exposure time $251 \mathrm{~ms}$, frame averaging 2, beam filtration filter $1 \mathrm{~mm}$ aluminum. After scanning, the knee joints were three-dimensionally reconstructed by SkyScan ReCon software (13). For histological analysis, the same knee joints were decalcified at $4{ }^{\circ} \mathrm{C}$ for $4-5$ weeks with $0.5 \mathrm{M}$ ethylenediaminetetraacetic acid (EDTA). The samples were serially dehydrated with successive changes with ethanol and embedded in paraffin by the standard method (14). 5-7 $\mu \mathrm{m}$ thick serial sections of the femur were prepared in the sagittal plane, stained with hematoxylin and eosin $(\mathrm{H} \& \mathrm{E})$ and the images were captured using Nikon Eclipse TE2000-S Microscope (Japan).

\section{Statistical analysis}

Statistical analysis was carried out using ANOVA to determine the significant differences between groups, followed by Student's Newman-Keul's test using GraphPad Prism 8.0.2 software. ${ }^{*} \mathrm{P}<0.05$ implies significance; ${ }^{a}$ : comparison with the control group; ${ }^{b}$ : comparison with OA group.

\section{Results}

The hPMSCs cultured in DMEM/F12 medium supplemented 10\% FBS exhibited spindle-shaped and fibroblastic morphology when viewed under a bright field microscope (Figure 1A). To evaluate the multi-lineage differentiation potential of the hPMSCs, the culture expanded hPMSCs were differentiated into osteogenic lineage as evaluated by Alizarin Red staining (Figure 1B), chondrogenic lineage as assessed by Alcian Blue Staining (Figure $1 C$ ) and adipogenic lineage as evaluated by Oil Red $\mathrm{O}(\mathrm{ORO})$ Staining (Figure 1D) when cultured in appropriate differentiation conditions. This confirmed the multilineage differentiation potential of the isolated hPMSCs. Immunofluorescence studies revealed that the hPMSCs stained positive for CD133 and STRO-1 which are well established stem cell markers (Figure 1E).

Micro-CT studies indicated that the control rats exhibited a smooth joint tissue surface with normal tissue architecture (Figure $2 A$ ). In the MIA-induced OA rats (Group II-A), micro-CT studies revealed severe erosion of the femoral condyle and trochlear groove cartilage, exposing the subchondral cancellous bone (Figure $2 B$ ). In OA rats treated with hPMSCs (Group II-B), there was a minimal repair/regenerative effect evident in the femoral condyles (Figure 2C). Little or no ameliorative effects were visualized by micro-CT in the knee joints of OA rats treated with stigmasterol per se (Group II-C) (Figure 2D). Notably, the OA rats treated with a combination of hPMSCs and Stigmasterol (Group II-D) showed a very significant tissue repair/regeneration detected by the concealment of the medial and lateral femoral condyles as well as the trochlear groove which was comparatively smoother with the masking of subchondral cancellous bone (Figure $2 E$ ).

Histological evaluation of the knee joints of the control and experimental rats confirmed the evidence obtained by micro-CT analysis. Control rats exhibited a smooth and healthy cartilage surface with no detectable abnormalities (Figure 3A). The MIA-induced OA rats (Group II-A) showed a very severe erosion of the articular cartilage and the subchondral bone leaving a 'hollow' at the end of the femur (Figure 3B). OA rats treated with hPMSCs (Group II-B) showed minimal cartilage regeneration (Figure $3 C$ ), while the OA rats treated with Stigmasterol (Group II-C) showed little/no reparative effect in the cartilage (Figure 3D). $\mathrm{OA}$ rats treated with a combination of hPMSCs and stigmasterol (Group II-D) showed a very significant cartilage regeneration/repair compared against all other treatment groups, emphasizing the benefit of the combination therapy over the individual treatments (Figure 3E). The Osteoarthritis Research Society International (OARSI) grading for cartilage histopathology also revealed that 

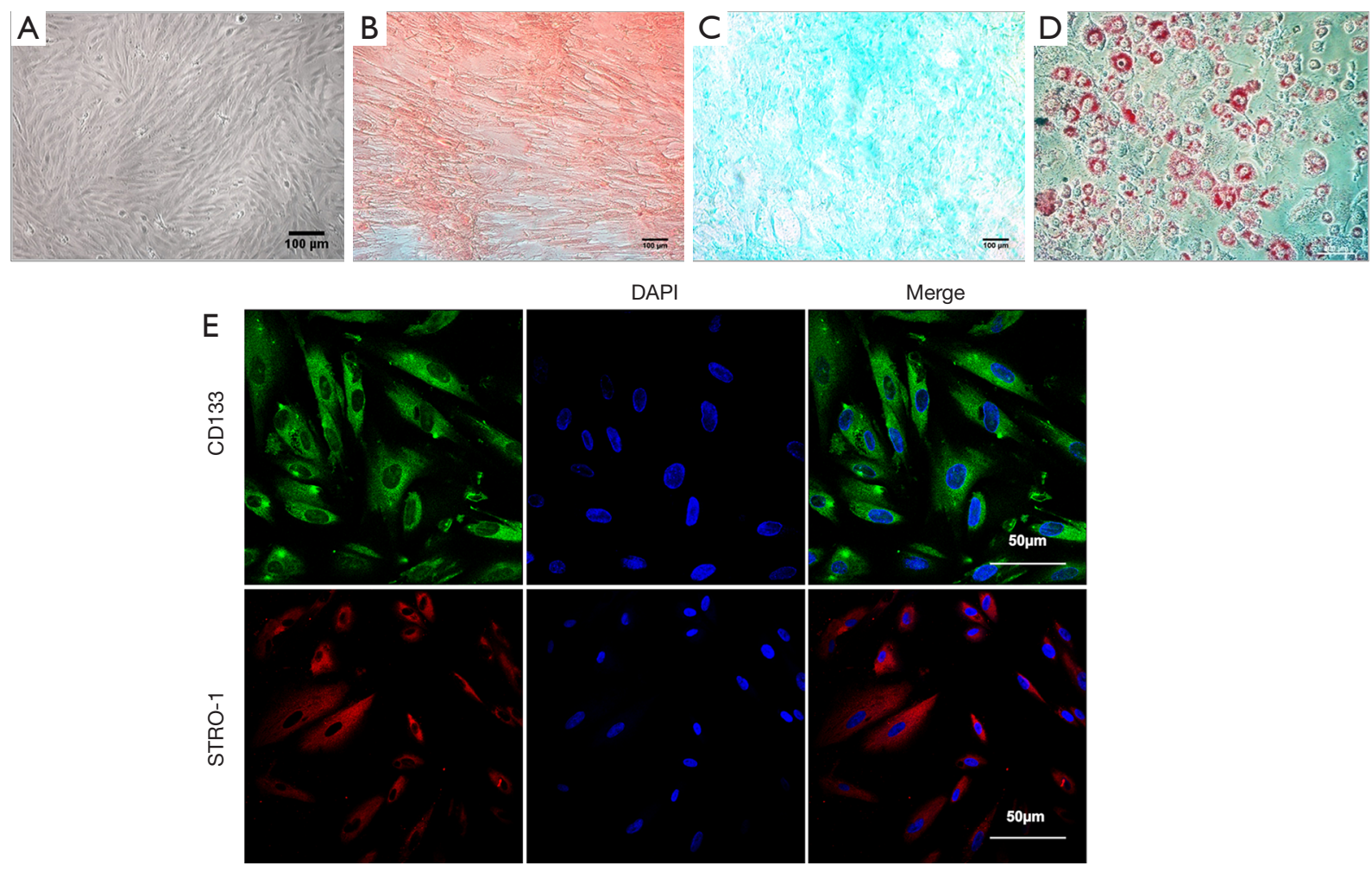

Figure 1 Characterization of human placental derived mesenchymal stem cells (hPMSCs). (A) hPMSCs micrograph under a bright-field microscope. (B) Alizarin Red Staining for osteogenic differentiation. (C) Alcian Blue staining for chondrogenic differentiation. (D) Oil-red - O staining for adipogenic differentiation. (E) Confocal micrograph for CD133 expression (top panel) and STRO-1 expression (bottom panel) in hPMSCs (P3). hPMSCs, human placental-derived mesenchymal stem cells.

there was a significant difference in OARSI scores in all the groups compared to the control group $(\mathrm{P}<0.05)$. No significant differences in OARSI scores were found in hPMSC treated (Group II-B) and stigmasterol treated (Group II-C) OA rats when compared with OA group (Group II-A). However, a significant improvement $(\mathrm{P}<0.05)$ in the OARSI score was found in the OA rats treated with the hPMSCs+stigmasterol combination therapy (Group IID) compared to the OA group (Group II-A) (Figure 3F).

\section{Discussion}

$\mathrm{OA}$ is the most common joint disorder that primarily affects the diarthrodial joints and progresses into severe degenerative arthritis, leading to debilitating conditions. Several non-pharmacological and pharmacological approaches including non-steroidal anti-inflammatory drugs (NSAIDs), analgesics, intra-articular corticosteroids, hyaluronic acid and dietary supplements have been employed to manage OA mainly focusing on alleviating pain, improving joint function and slowing down joint damage (15). To this effect, studies carried out in clinical settings on OA patients have also observed that combining therapeutic agents with complementing mechanisms of actions resulted in better efficacy in reducing pain, inflammation and improving joint function when compared to the individual treatments (16-19). However, all these therapeutic options fail to regenerate the lost cartilage during OA progression. Hence, in the current study, we evaluated the beneficial effects of combining MSCs with stigmasterol in inducing cartilage repair/regeneration in the chemically induced rat OA model.

In the current study, MIA injection resulted in severe cartilage erosion closely mimicking the human OA pathology. Gabay et al. [2010] earlier demonstrated that stigmasterol inhibited key pro-inflammatory and matrix degradation mediators [interleukin-6 (IL-6), MMPs-3 \& 13, A Disintegrin and Metalloproteinase with Thrombospondin motifs (ADAMTS)-4 and prostaglandin E-2 (PGE2)] involved in OA-induced cartilage degradation, 

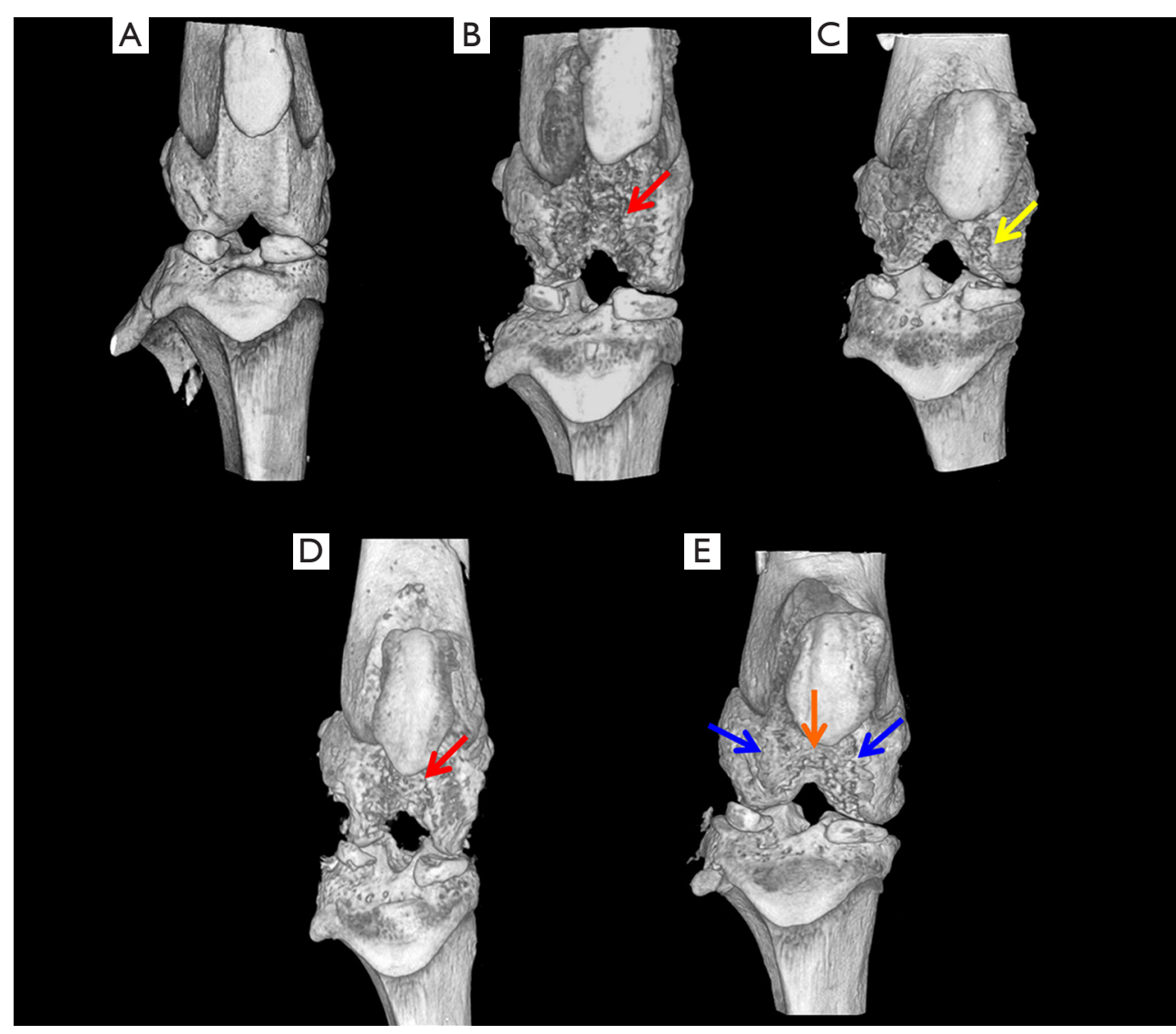

Figure 2 Three-dimensional micro-CT evaluation of control and experimental rat knee joints. (A) Group I: control rats. (B) Group (II-A): MIA-induced OA rats (red arrow indicates erosion). (C) Group (II-B): OA rats treated with hPMSCs (yellow arrow indicates minimal tissue repair). (D) Group (II-C): OA rats treated with stigmasterol (red arrow indicates little/no repair) (E) Group (II-D): OA rats treated with hPMSCs and stigmasterol revealed tissue repair at femoral condyles (blue arrows) and trochlear groove (orange arrow). MIA, monosodiumiodoacetate; OA, osteoarthritis; hPMSCs, human placental-derived mesenchymal stem cells.

predominantly by inhibiting the NF-אB pathway (6). In an anterior cruciate ligament transection (ACLT) rabbit model of surgical OA, Chen et al. [2012] have shown that intraarticular injection of stigmasterol significantly reduced cartilage degradation in rabbits as observed by gross morphology, histology and suppression of MMP-1, 3 and 13 (7). In our current study, we couldn't recapitulate the chondroprotective effects of stigmasterol per se, as reported by Chen et al., essentially due to the difference in the method for inducing OA in the animal models. While the hPMSCs per se did seem to offer some promise in cartilage regeneration in the OA rats (Group II-B), the outcome of the treatment was not noteworthy. The combination therapy (Group II-D) resulted in a significant repair/ regeneration of the eroded cartilage in the femoral condyles as well as the trochlear groove, accentuating the promise of the combination therapy over the individual treatments in the OA rats.

The results of our study showed that the combined administration of hPMSCs with stigmasterol was the most effective in correcting the OA lesions, with concomitant repair and regeneration, providing valuable insights in situ using a combination of hPMSCs and stigmasterol towards enhanced cartilage repair and regeneration in OA. We advocate that the intra-articularly injected hPMSCs could have homed and engrafted to the chondral lesions in the MIA-injected knee joints and produced cartilage matrix to bring about repair via tissue regeneration. Also, the paracrine secretions of MSCs could have provided a suitable milieu in situ and aided in cartilage regeneration by activating the resident progenitors to enhance tissue repair and healing. Combining hPMSCs with stigmasterol 

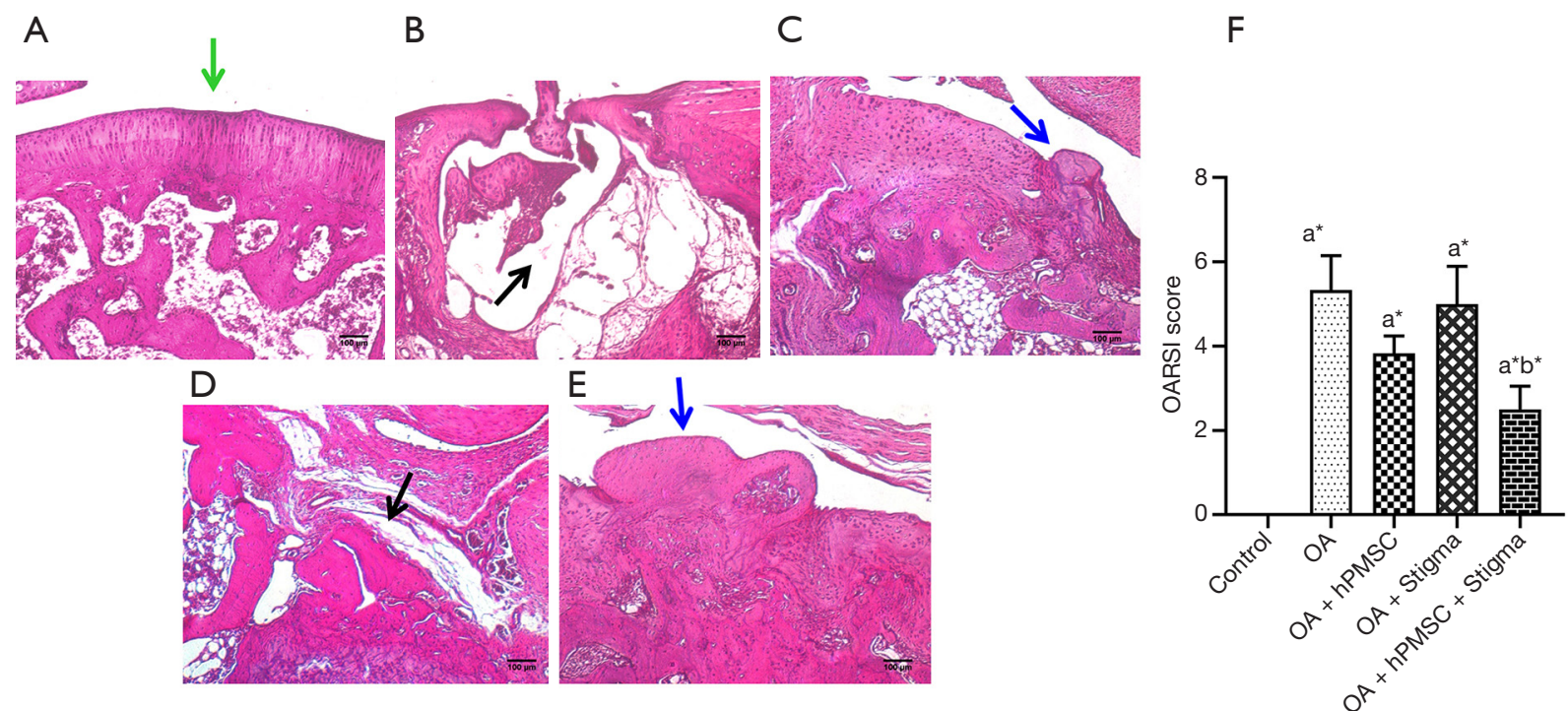

Figure 3 Histological evaluation of femur in control and experimental rats by haematoxylin \& eosin staining. (A) Group I: control rats (green arrow indicates a smooth cartilage surface). (B) Group (II-A): OA rats (black arrow indicates cartilage erosion). (C) Group (II-B): OA rats treated with hPMSCs (blue arrow indicates minimal cartilage regeneration). (D) Group (II-C): OA rats treated with stigmasterol (black arrow indicates little/no cartilage repair). (E) Group (II-D): OA rats treated with a combination of hPMSCs and stigmasterol (blue arrow indicates a very significant cartilage regeneration/repair). (F) OARSI grading for cartilage histopathology. Values are indicated as mean \pm SD ( $\mathrm{n}=6)$. Statistical analysis was carried out using ANOVA to determine the significant differences between groups, followed by Student's Newman-Keul's test. *, $\mathrm{P}<0.05$ implies significance, ${ }^{\mathrm{a}}$, comparison with the control group; ${ }^{\mathrm{b}}$, comparison with OA group. OA, osteoarthritis; hPMSCs, human placental-derived mesenchymal stem cells.

could have enhanced cross-talk between the two different treatment modalities and possibly augmented the chondrogenic differentiation potential of the injected hPMSCs, thereby promoting cartilage regeneration and repairing the erosion at the site of injury.

So far, many clinical trials have been carried out using human OA subjects intending to evaluate the employability of MSCs/stromal cells as 'cell therapy' for OA. A majority of these studies have shown successful cartilage renewal and pain relief. In this context, the novel finding of our study offers immense promise for the combination therapy to have a synergistic effect in addressing generalized cartilage loss in OA and bring about better disease modification. Stigmasterol with inherent anti-inflammatory and chondroprotective properties combined with hPMSCs possessing immunomodulatory, anti-inflammatory and multipotent functions has definite advantages in promoting chondrogenesis vis a vis cartilage regeneration in OA. The findings of our study add impetus to the promise offered by MSCs in tissue engineering and regenerative medicine especially in the field of OA with a more prolonged benefit. Studies at the molecular level would unravel the exact mechanisms by which the combination therapy of hPMSCs and stigmasterol bring about cartilage regeneration, which are underway.

\section{Acknowledgments}

Funding: This work was supported by financial support by the Indian Council of Medical Research, New Delhi to Sampath Samuel Joshua Pragasam through postdoctoral research fellowship (Grant. No. (3/1/3/PDF (14)/2016HRD). The authors also thank the Director of the Institute and the Animal Facility for infrastructure support.

\section{Footnote}

Conflicts of Interest: All authors have completed the ICMJE uniform disclosure form (available at http://dx.doi. org/10.21037/sci-2020-048). The authors have no conflicts of interest to declare.

Ethical Statement: The authors are accountable for all aspects of the work in ensuring that questions related 
to the accuracy or integrity of any part of the work are appropriately investigated and resolved. All animal experiments were carried out in accordance with the guidelines of the Committee for the Purpose of Control and Supervision of Experiments on Animals (CPCSEA). The study was approved by the Institutional Animal Ethical Committee, National Institute of Nutrition, Hyderabad (P29F/III-IAEC/NIN/12/2016).

Open Access Statement: This is an Open Access article distributed in accordance with the Creative Commons Attribution-NonCommercial-NoDerivs 4.0 International License (CC BY-NC-ND 4.0), which permits the noncommercial replication and distribution of the article with the strict proviso that no changes or edits are made and the original work is properly cited (including links to both the formal publication through the relevant DOI and the license). See: https://creativecommons.org/licenses/by-nc-nd/4.0/.

\section{References}

1. Goldring MB, Otero M, Plumb DA, et al. Roles of inflammatory and anabolic cytokines in cartilage metabolism: signals and multiple effectors converge upon MMP-13 regulation in osteoarthritis. Eur Cell Mater 2011;21:202-20.

2. Zhang $W$, Robertson $W B$, Zhao J, et al. Emerging Trend in the Pharmacotherapy of Osteoarthritis. Front Endocrinol (Lausanne) 2019;10:431.

3. Fan XL, Zhang Y, Li X, et al. Mechanisms underlying the protective effects of mesenchymal stem cell-based therapy. Cell Mol Life Sci 2020;77:2771-94.

4. Wang M, Yuan Q, Xie L. Mesenchymal Stem Cell-Based Immunomodulation: Properties and Clinical Application. Stem Cells Int 2018;2018:3057624.

5. Chaudhary J, Jain A, Kaur N, et al. Stigmasterol: A Comprehensive Review. Int J Pharm Sci Res 2011;2:2259.

6. Gabay O, Sanchez C, Salvat C, et al. Stigmasterol: a phytosterol with potential anti-osteoarthritic properties. Osteoarthritis Cartilage 2010;18:106-16.

7. Chen WP, Yu C, Hu PF, et al. Stigmasterol blocks cartilage degradation in rabbit model of osteoarthritis. Acta Biochim Pol 2012;59:537-41.

8. Sundararaman P, Djerassi C. A convenient synthesis of progesterone from stigmasterol. J Org Chem 1977;42:3633-4.

9. Woo YJ, Joo YB, Jung YO, et al. Grape seed proanthocyanidin extract ameliorates monosodium iodoacetate-induced osteoarthritis. Exp Mol Med 2011;43:561-70.

10. Kadam S, Muthyala S, Nair P, et al. Human placentaderived mesenchymal stem cells and islet-like cell clusters generated from these cells as a novel source for stem cell therapy in diabetes. Rev Diabet Stud 2010;7:168-82.

11. Madhira SL, Challa SS, Chalasani M, et al. Promise(s) of mesenchymal stem cells as an in vitro model system to depict pre-diabetic/diabetic milieu in WNIN/GR-Ob mutant rats. PLoS One 2012;7:e48061.

12. Bar-Yehuda S, Rath-Wolfson L, Del Valle L, et al. Induction of an Antiinflammatory Effect and Prevention of Cartilage Damage in Rat Knee Osteoarthritis by CF101 Treatment. Arthritis Rheum 2009;60:3061-71.

13. Wang T, Wen CY, Yan CH, et al. Spatial and temporal changes of subchondral bone proceed to microscopic articular cartilage degeneration in guinea pigs with spontaneous osteoarthritis. Osteoarthritis Cartilage 2013;21:574-81.

14. Pragasam SJ, Murunikkara V, Sabina EP, et al. Ameliorative effect of p-coumaric acid, a common dietary phenol, on adjuvant-induced arthritis in rats. Rheumatol Int 2013;33:325-34.

15. Yusuf E. Pharmacologic and Non-Pharmacologic Treatment of Osteoarthritis. Curr Treatm Opt Rheumatol 2016;2:111-25.

16. Lubis AMT, Siagian C, Wonggokusuma E, et al. Comparison of Glucosamine-Chondroitin Sulfate with and without Methylsulfonylmethane in Grade I-II Knee Osteoarthritis: A Double Blind Randomized Controlled Trial. Acta Med Indones 2017;49:105-11.

17. Yu W, Xu P, Huang G, et al. Clinical therapy of hyaluronic acid combined with platelet-rich plasma for the treatment of knee osteoarthritis. Exp Ther Med 2018;16:2119-25.

18. Stitik TP, Blacksin MF, Stiskal DM, et al. Efficacy and safety of hyaluronan treatment in combination therapy with home exercise for knee osteoarthritis pain. Arch Phys Med Rehabil 2007;88:135-41.

19. Zeng C, Wei J, Li H, et al. Effectiveness and safety of Glucosamine, chondroitin, the two in combination, or celecoxib in the treatment of osteoarthritis of the knee. Sci Rep 2015;5:16827.

doi: $10.21037 /$ sci-2020-048

Cite this article as: Sampath SJP, Kotikalapudi N, Venkatesan V. A novel therapeutic combination of mesenchymal stem cells and stigmasterol to attenuate osteoarthritis in rodent model system-a proof of concept study. Stem Cell Investig 2021;8:5. 